\title{
Memory and Electrical Properties of (100)-Oriented AlN Thin Films Prepared by Radio Frequency Magnetron Sputtering
}

\author{
Maw-Shung Lee, ${ }^{1}$ Sean Wu, ${ }^{2}$ Shih-Bin Jhong, ${ }^{1}$ Kai-Huang Chen, ${ }^{3}$ and Kuan-Ting Liu ${ }^{4}$ \\ ${ }^{1}$ Department of Electronics Engineering, National Kaohsiung University of Applied Sciences, Kaohsiung 807, Taiwan \\ ${ }^{2}$ Department of Electronics Engineering and Computer Science, Tung Fang Design Institute, Kaohsiung 829, Taiwan \\ ${ }^{3}$ Department of Animation and Multimedia Design, Tung Fang Design Institute, Kaohsiung 829, Taiwan \\ ${ }^{4}$ Department of Electronics Engineering, Cheng-Shiu University, Kaohsiung 833, Taiwan
}

Correspondence should be addressed to Sean Wu; wusean.tw@gmail.com

Received 19 June 2014; Accepted 18 July 2014; Published 11 September 2014

Academic Editor: Teen-Hang Meen

Copyright (C) 2014 Maw-Shung Lee et al. This is an open access article distributed under the Creative Commons Attribution License, which permits unrestricted use, distribution, and reproduction in any medium, provided the original work is properly cited.

\begin{abstract}
The (100)-oriented aluminum nitride (AlN) thin films were well deposited onto p-type Si substrate by radio frequency (RF) magnetron sputtering method. The optimal deposition parameters were the RF power of $350 \mathrm{~W}$, chamber pressure of $9 \mathrm{mTorr}$, and nitrogen concentration of 50\%. Regarding the physical properties, the microstructure of as-deposited (002)- and (100)-oriented AlN thin films were obtained and compared by XRD patterns and TEM images. For electrical properties analysis, we found that the memory windows of (100)-oriented AlN thin films are better than those of (002)-oriented thin films. Besides, the interface and interaction between the silicon and (100)-oriented AlN thin films was serious important problem. Finally, the current transport models of the as-deposited and annealed (100)-oriented AlN thin films were also discussed. From the results, we suggested and investigated that large memory window of the annealed (100)-oriented AlN thin films was induced by many dipoles and large electric field applied.
\end{abstract}

\section{Instructions}

Recently, the various functional thin films were widely focused on the applications in nonvolatile random access memory (NvRAM), such as smart cards and portable electrical devices, utilizing excellent memory characteristics, high storage capacity, long retention cycles, low electric consumption, nonvolatility, and high speed readout. Additionally, the various nonvolatile random access memory devices such as ferroelectric random access memory (FeRAM), magnetron memory (MRAM), resistance random access memory (RRAM), and flash memory were widely discussed and investigated [1-9]. However, the high volatile pollution elements and high fabrication cost of the complex composition material were serious difficult problems for applications in integrated circuit semiconductor processing. For this reason, the simple binary metal oxide materials such as $\mathrm{ZnO}, \mathrm{Al}_{2} \mathrm{O}_{3}$, $\mathrm{TiO}_{2}$, and $\mathrm{Ta}_{2} \mathrm{O}_{5}$ were widely considered and investigated for the various functional electronic product applications in resistance of random access memory devices [10-12].
The AlN thin film was promising piezoelectric materials for GHz-band surface acoustic wave SAW devices because of its high ultrasonic velocity, piezoelectricity, and thermal stability. In addition, AlN thin films exhibited (002)-, (100)-, and (103)-oriented hexagonal wurtzite structure and wide band gap in the range 5.9-6.2 eV [13]. For the ultrasonic velocity properties, the coupling coefficient was an important parameter and was chosen for the SAW devices. According to previous study, the coupling coefficient of the (100)-oriented AlN thin films is larger than the (002)-oriented thin films. However, it was difficult to prepare the (100)-oriented AlN thin films by PVD method $[14,15]$.

In this report, the subject of this work was to study the possible application of (100)-oriented AlN thin films deposited onto p-type silicon substrate using the RF magnetron sputtering. In addition, the physical and electrical characteristics of (100)-oriented AlN thin films were well developed and investigated in the metal-insulatorsemiconductor (MIS) structure. The leakage current density, current transport mechanism, and the memory window of 


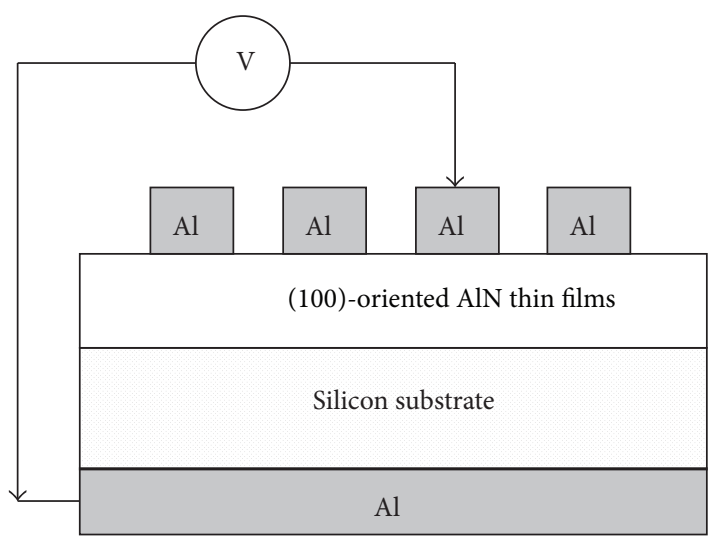

FIGURE 1: Metal insulator semiconductor (MIS) structure using asdeposited (100)-oriented AlN thin films.

(100)-oriented AlN thin films will be characterized. We will show that (100)-oriented AlN thin film is a candidate for fabrication of the memory devices.

\section{Experimental Details}

Metal insulator semiconductor (MIS) device, schematically shown in Figure 1, was fabricated over a polished p-Si wafer of nominal resistively $\sim 1.0 \mathrm{ohm}-\mathrm{cm}$. To overcome the existence of native-oxide on p-type silicon wafer, the particle and metal ion on p-type silicon wafer were removed during RCA clean process. The Al target was placed near $9.5 \mathrm{~cm}$ away from the substrate. The AlN thin films were deposited on a ptype $\mathrm{Si}$ substrate by RF magnetron sputtering method. The thin films were deposited on silicon substrate at $200^{\circ} \mathrm{C}$ under the optimal deposition parameters such as the RF power of $350 \mathrm{~W}$, chamber pressure of 9 mTorr, and nitrogen concentration of $50 \%$. The XRD patterns of the AlN thin films were recorded to find the crystallographic structure of thin film in the $2 \theta$-degree range of $20^{\circ} \sim 60^{\circ}$ using SIEMENS D5000. To complete the MIS structure, an array of circular top contacts with a diameter of $0.1 \mathrm{~cm}$ was formed by depositing an Al film with thickness of $500 \mathrm{~nm}$ through a metal mask by thermal evaporation in a vacuum system operating at $1 \times 10^{-5}$ Torr. The capacitance and leakage of current characteristics of AlN thin films were measured by a gain phase analyzer (HP 4194A) and a semiconductor parameter analyzer (HP 4156).

\section{Results and Discussion}

To determine and investigated the difference preferred phase on AlN thin films in this study, the XRD pattern of the (100)and (002)-oriented AlN thin films was studied and discussed. According to a few reports; the (100) and (001) preferential orientation of AlN thin films were controlled and prepared, because electromechanical coupling coefficient of the (100)oriented thin films was suitable for that of the (001)-oriented thin films. However, it was difficult to prepare the (100)oriented AlN thin films by sputtering and other technologies $[14,15]$. Ishihara et al. already reported that the (100) and

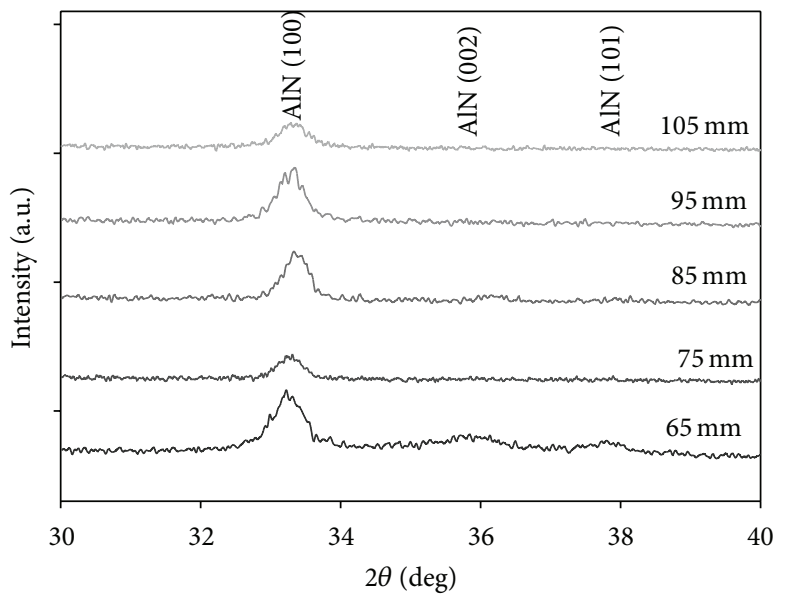

FIGURE 2: The XRD patterns of as-deposited (100)-oriented AlN thin films for different sputtering distance.

(001) preferential orientation of AlN films were prepared and controlled by the reactive sputtering method. A long distance between the $\mathrm{Al}$ target and substrate and the high sputtering pressures prepared and deposited for (100)-oriented AlN thin films were also found and discussed [13].

Figure 2 shows X-ray diffraction patterns of (100)oriented AlN thin films for different distance between the $\mathrm{Al}$ target and substrate on a p-type silicon substrate. From the XRD pattern, we found that the AlN thin films exhibited polycrystalline structure. All of AlN thin films exhibited well $a$-axis orientation. According to Joint Committee for Powder Diffraction Studies (JCPDS) card of AlN material (number 25-1133), the (100), (002), and (101) peaks were observed in the XRD pattern. For the distance of $95 \mathrm{~mm}$, the AlN thin films exhibited the preferred (100) orientation. At the distance of $95 \mathrm{~mm}$, the intensity of the AlN (002) and (101) peak disappeared and the peaks of the AlN (100) appear. We found and suggested that (100)-oriented AlN thin films were prepare by making the suitable distance from the experimental results. The optimal deposition conditions such as the RF power of $350 \mathrm{~W}$, chamber pressure of $9 \mathrm{mTorr}$, and nitrogen concentration of $50 \%$. In addition, the $2 \theta$ diffraction peak of (101)- and (002)-oriented AlN thin film were $37.925^{\circ}$ and $36.039^{\circ}$, respectively. In addition, $d_{101}$ and $d_{002}$ lattice constant of the AlN thin films were 0.2371 and $0.249 \mathrm{~nm}$.

The high resolution transmission electron microscopy (HRTEM) image shows the (100)-oriented structure in the AlN thin films, as shown in Figure 3. The thickness of (100)oriented AlN thin films was about $1 \mu \mathrm{m}$ in Figure 3(a). The $d_{100}$ lattice constant of the (100)-oriented AlN thin films was calculated and it was about $0.27 \mathrm{~nm}$ using the following Bragg diffraction equation:

$$
n \lambda=2 d \cdot \sin \theta
$$

where $n$ is the diffraction time. The $\lambda$ is the wavelength of the X-ray diffraction. The $\theta$ is injection angle of the Xray diffraction. The $d$ is the spacing of lattice. Therefore, these results proved that the AlN thin films exhibited (100)oriented structure in Figure 3(b). 


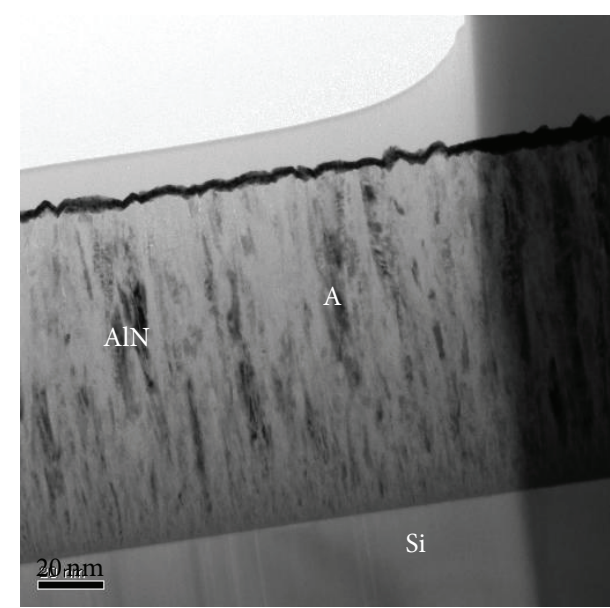

(a)

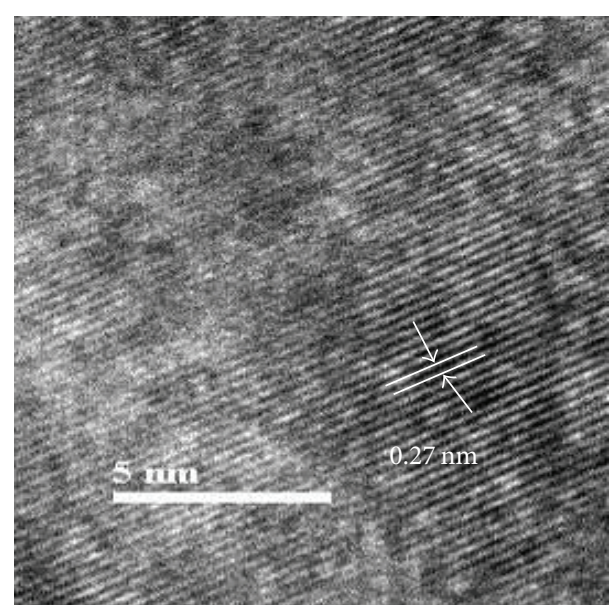

(b)

FIgURE 3: The (a) cross-sectional images and (b) $d_{100}$ lattice distance of as-deposited (100)-oriented AlN thin films.

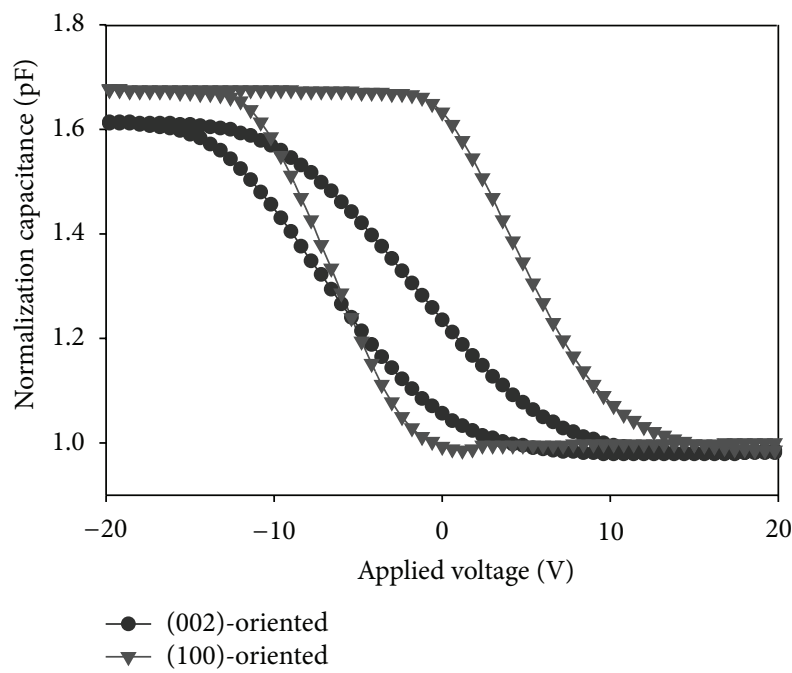

FIGURE 4: The normalization capacitance versus applied voltage curves of as-deposited (002)- and (100)-oriented AlN thin films.

Figure 4 shows the normalization capacitance versus the applied voltage $(C-V)$ curves of the (100)- and (002)-oriented AlN thin films in MIS structure measured at $1 \mathrm{MHz}$. The applied voltages, which were first changed from $-20 \mathrm{~V}$ to $20 \mathrm{~V}$ and then returned to $-20 \mathrm{~V}$, were used to measure the capacitance voltage characteristics $(C-V)$ curves. We found that the normalization capacitances of (002)- and (100)-oriented AlN thin films were 1.61 and $1.67 \mathrm{pF}$, respectively. However, the (100)-oriented AlN thin films exhibited high normalization capacitance than those of (002)-oriented AlN thin films. As shown in Figure 4, the normalization capacitances of the (100)-oriented AlN thin films appear to increase due to its $a$-axis orientation structure. From the experimental results obtained, the oxide trap charges $\left(Q_{\mathrm{ot}}\right)$ number was calculated and found using the following:

$$
Q_{\mathrm{ot}}=\frac{\Delta V_{\mathrm{FB}} \cdot C}{q \cdot A},
$$

where the $C$ and $A$ value are capacitance and area of the AlN capacitor, respectively. The elementary charge $q=1.60218 \times$ $10^{-19} \mathrm{C}$. The $\Delta V_{\mathrm{FB}}$ is the flat-band voltage. The $Q_{\mathrm{ot}}$ is the oxide trap charges numbers.

As shown in Figure 4, the $Q_{\text {ot }}$ number for the asdeposited (100)-oriented AlN thin films was $1 \times 10^{3}$. Additionally, the threshold voltage of (002)- and (100)-oriented AlN thin films was $10 \mathrm{~V}$ and $13 \mathrm{~V}$, respectively. We suggested that the high threshold voltage of AlN thin films for (100)-oriented structure caused by many defects and vacancies in $C-V$ curves. In a previous study, the threshold voltage of dielectric thin films was attributed by defects and vacancies [9]. These $C-V$ experimental results were proved and discussed from the $J E$ properties of AlN thin films. In addition, the memory window of the (002)- and (100)-oriented AlN thin films was observed and found. They were about $10 \mathrm{~V}$ and $13 \mathrm{~V}$, respectively. The memory window of (100)-oriented AlN thin films was larger than those (002)-oriented thin films. In addition, the memory window of (100)-oriented AlN thin films was changed by the sweeping speed in Figure 5. The threshold voltage of (100)-oriented AlN thin films in MIS structure decreases as the sweeping speed increases. That was influenced by the mobile ions and charge injection between as-deposited (100)-oriented AlN thin films and metal electrode as the sweeping speed increased [9].

For MIS structure, the interface and interaction between the silicon and dielectric films was serious important problem. From the leakage current density versus electrical field $(J E)$ curves, the conduction mechanism of as-deposited (002)- and (100)-oriented AlN thin films also proved the defect and vacancy results. Figure 6 shows the $J E$ characteristics of as-deposited (100)-oriented AlN thin films for the different deposited time. The leakage current density of the as-deposited AlN thin films for $1 \mathrm{hr}$ deposited time was lower than those of other thin films. The few defects and vacancies were also proved and found from the $Q_{\text {ot }}$ charges number of the as-deposited AlN thin films for $1 \mathrm{hr}$ time in $C-V$ curves. To discuss the oxide charge $Q_{\text {ot }}$ effect, the leakage current versus 


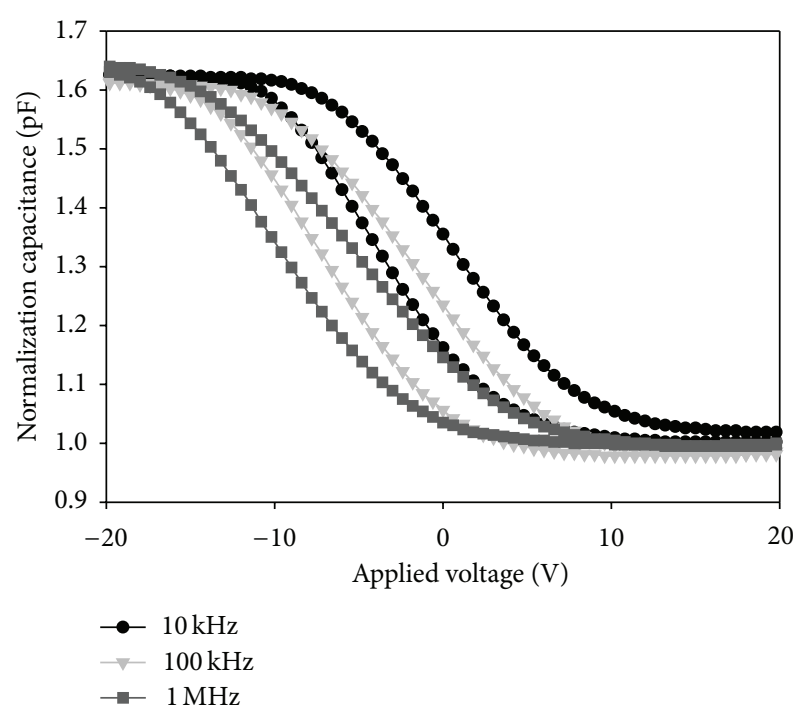

FIGURE 5: The normalization capacitance versus applied voltage curves of as-deposited (100)-oriented AlN thin films for different sweeping speed.

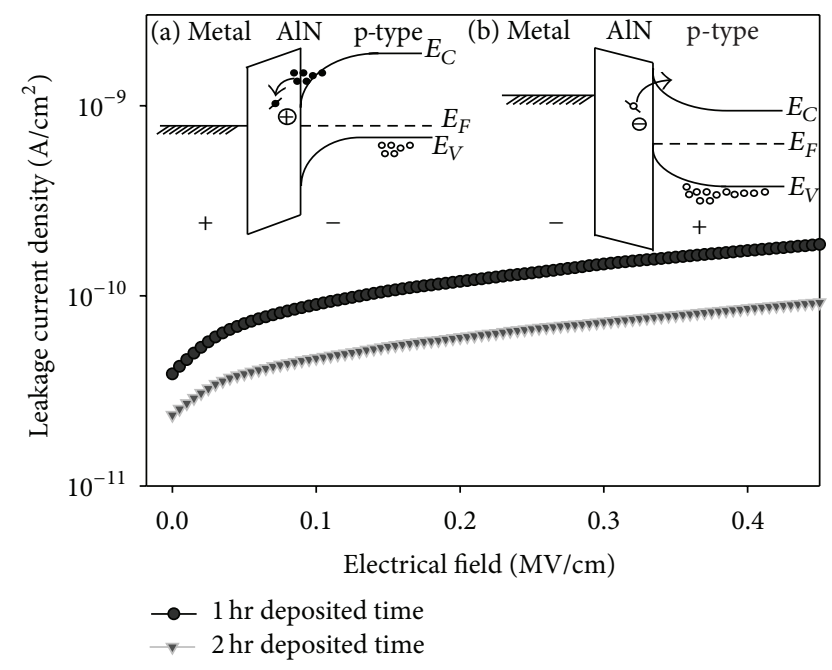

FIGURE 6: The leakage current versus applied voltage characteristics of as-deposited (100)-oriented AlN thin films for the different deposited time.

electrical field curves of the as-deposited AlN thin films were fitted to Schottky emission, Poole-Frankel, and space charge limit conduction (SCLC) transport models. As shown in insert of Figure 6, the schematic energy band diagrams under positive and negative bias conditions were discussed and found, respectively. For positive bias, the electrons were attracted from the p-type Si substrate to the as-deposited (100)-oriented AlN thin films and captured by deep traps of the AlN thin films (Figure 6(a)). For negative bias, the trapped electrons of as-deposited AlN thin films emit from the traps to the p-type Si substrate, corresponding to the left shift of the $C-V$ curve (Figure 6(b)). The large memory window of the as-deposited AlN thin films might be caused by defects and vacancies.

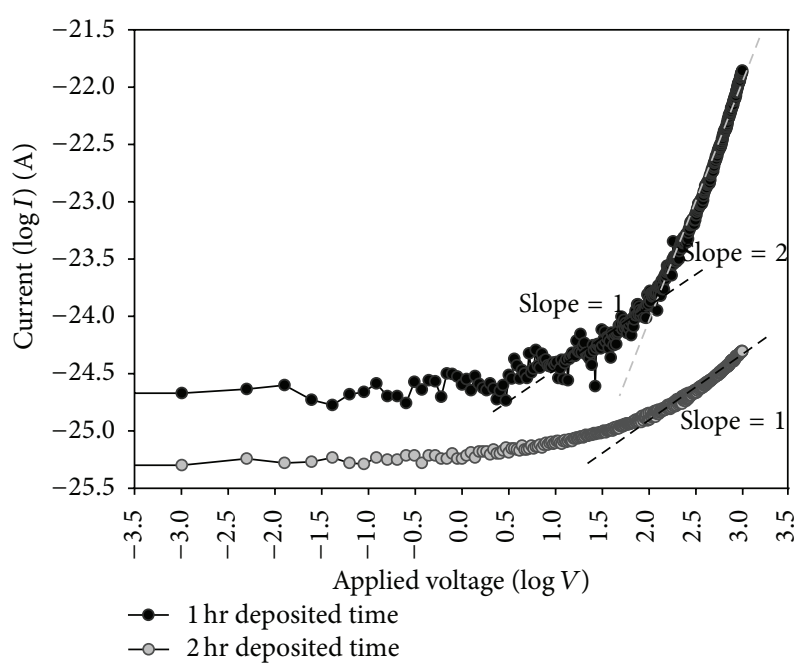

FIGURE 7: The leakage current versus applied voltage characteristics of as-deposited (100)-oriented AlN thin films for the different deposited time.

Figure 7 shows the $J E$ characteristics for the as-deposited AlN thin films in terms of $\log I$ as vertical axis and $\log V$ as horizontal axis. The fitting curves were straight and had different slope in this figure and $J E$ curve of AlN thin films was the SCLC theory [16].

The child's law equation of space charge limit conduction is as follows:

$$
J_{\text {child }}=\frac{9}{8} \varepsilon \mu_{n} \frac{V^{2}}{d_{s}^{3}},
$$

where the $\varepsilon, \mu_{n}$, and $d_{s}$ value of the AlN capacitor are dielectric constant, electronic mobility, and thickness, respectively. For the slope $=1$, the as-deposited AlN thin films exhibited the ohmic conduction in low applied voltage, and it was from $0.7 \mathrm{~V}$ to $3 \mathrm{~V}$. For the slope $=2$, the as-deposited AlN thin films exhibited child's law in high applied voltage and it was trap center region. The results were proved and caused by the carrier filling the trap and defect of the as-deposited AlN thin films. However, the as-deposited AlN thin films for $2 \mathrm{hr}$ time did not exhibited the child's law in high applied voltage applied. The high leakage current was also attributed to the vacancies and defects of as-deposited thin films.

Figure 8 shows the same $J E$ characteristics in terms of $J / T^{2}$ as vertical axis and applied electrical filed $E^{1 / 2}$ as horizontal axis. The fitting curves were straight in this figure, and the $J E$ curves obey the Schottky emission model [1719]. For as-deposited thin films, the JE current of AlN thin films was fitted well by straight lines in low applied electrical filed. According to fitting curves in $J E$ current, we found and suggested that the as-deposited AlN thin films exhibited the Schottky emission model in high applied electrical filed. Additionally, we found that the as-deposited AlN thin films for $2 \mathrm{hr}$ deposited time only exhibited the Schottky emission model. In this study, the as-deposited (100)-oriented AlN thin films exhibited excellent memory and electrical properties were found. 


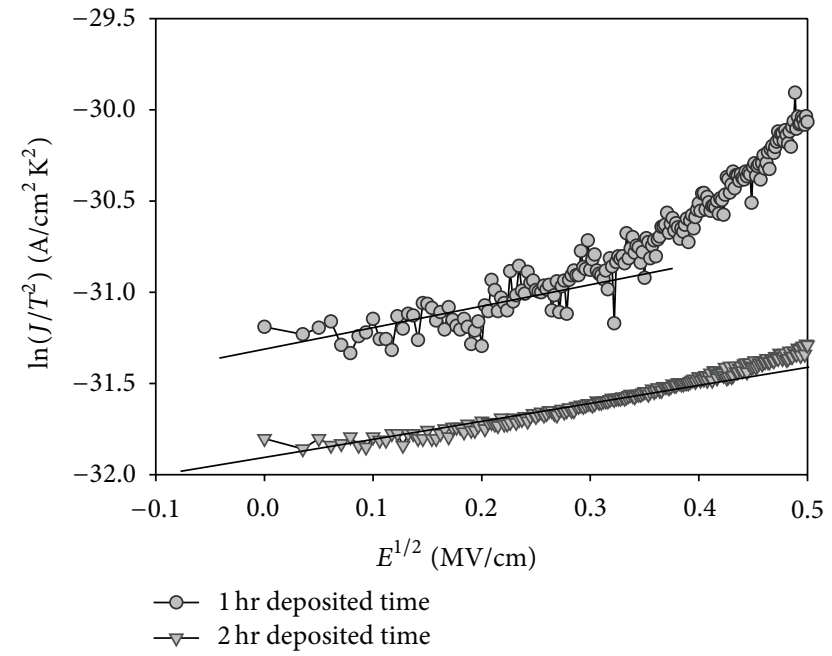

Figure 8: The $\left(J / T^{2}\right)$ versus $E^{1 / 2}$ curves of as-deposited (100)oriented AlN thin films for the different deposited time.

\section{Conclusion}

In summary, the (100)-oriented AlN thin films were prepared by RF magnetron sputtering technology. From the XRD patterns, all oriented AlN thin films on p-type substrate crystallized well. The $d_{100}$ lattice constant of the (100)oriented AlN thin films was calculated and it was about $0.27 \mathrm{~nm}$ using the Bragg diffraction equation. From the $C$ $V$ curves, the $Q_{\text {ot }}$ charge number of the as-deposited (100)oriented AlN thin films was $1 \times 10^{3}$. The memory window effect, electrical properties, and depletion delay of the MIS structure may be caused by oxide charge of the as-deposited AlN thin films in $C-V$ curves. The conduction mechanism of as-deposited (100)-oriented AlN thin films also proved these results in $J E$ curves.

\section{Conflict of Interests}

The authors declare that there is no conflict of interests regarding the publication of this paper.

\section{Acknowledgment}

This work acknowledges the financial support of the Ministry of Science and Technology of the Republic of China (NSC 102-2221-E-151-054-).

\section{References}

[1] S. R. Shannigrahi and H. M. Jang, "Fatigue-free lead zirconate titanate-based capacitors for nonvolatile memories," Applied Physics Letters, vol. 79, p. 1051, 2001.

[2] S. K. Hong, C. W. Suh, C. G. Lee, S. W. Lee, E. Y. Hang, and N. S. Kang, "Protection of $\mathrm{SrBi}_{2} \mathrm{Ta}_{2} \mathrm{O}_{9}$ ferroelectric capacitors from hydrogen damage by optimized metallization for memory applications," Applied Physics Letters, vol. 77, p. 76, 2000.
[3] S. B. Xiong and S. Sakai, "Memory properties of $\mathrm{SrBi}_{2} \mathrm{Ta}_{2} \mathrm{O}_{9}$ thin films prepared on $\mathrm{SiO}_{2} / \mathrm{Si}$ substrates," Applied Physics Letters, vol. 75, p. 1613, 1999.

[4] J. S. Kim and S. G. Yoon, "High dielectric constant (Ba0.65Sr 0.35)(Ti0.41Zr0.59)O3 capacitors for Gbit-scale dynamic random access memory devices," Journal of Vacuum Science \& Technology B, vol. 18, pp. 216-220, 2000.

[5] T. B. Wu, C. M. Wu, and M. L. Chen, "Highly insulative barium zirconate-titanate thin films prepared by $\mathrm{rf}$ magnetron sputtering for dynamic random access memory applications," Applied Physics Letters, vol. 69, p. 2659, 1996.

[6] K. H. Chen, Y. C. Chen, C. F. Yang, and T. C. Chang, "Fabrication and characteristics of $\mathrm{Ba}\left(\mathrm{Zr}_{0.1}, \mathrm{Ti}_{0.9}\right) \mathrm{O}_{3}$ thin films on glass substrate," Journal of Physics and Chemistry of Solids, vol. 69, no. 2-3, pp. 461-464, 2008.

[7] C. F. Yang, K. H. Chen, Y. C. Chen, and T. C. Chang, "Fabrication of one-transistor-capacitor structure of nonvolatile TFT ferroelectric RAM devices using $\mathrm{Ba}\left(\mathrm{Zr}_{0.1} \mathrm{Ti}_{0.9}\right) \mathrm{O}_{3}$ gated oxide film," IEEE Transactions on Ultrasonics, Ferroelectrics and Frequency Control, vol. 54, no. 9, pp. 1726-1730, 2007.

[8] C. F. Yang, K. H. Chen, Y. C. Chen, and T. C. Chang, "Physical and electrical characteristics of $\mathrm{Ba}\left(\mathrm{Zr}_{0.1} \mathrm{Ti}_{0.9}\right) \mathrm{O}_{3}$ thin films under oxygen plasma treatment for applications in nonvolatile memory devices," Applied Physics A: Materials Science \& Processing, vol. 90, no. 2, pp. 329-331, 2008.

[9] K. H. Chen, Y. C. Chen, Z. S. Chen, C. F. Yang, and T. C. Chang, "Temperature and frequency dependence of the ferroelectric characteristics of $\mathrm{BaTiO}_{3}$ thin films for nonvolatile memory applications," Applied Physics A, vol. 89, no. 2, pp. 533-536, 2007.

[10] S. Wu, Z. X. Lin, M. S. Lee, and R. Ro, "Bulk acoustic wave analysis of crystalline plane oriented $\mathrm{ZnO}$ films," Applied Physics Letters, vol. 102, Article ID 084908, 2007.

[11] S. Lee, E. B. Song, S. Kim et al., "Impact of gate work-function on memory characteristics in Al2O3/HfOx/Al2O3/graphene charge-trap memory devices," Applied Physics Letters, vol. 100, Article ID 023109, 2012.

[12] Y. Yang, L. Jin, X. Ma, and D. Yang, "Low-voltage driven visible and infrared electroluminescence from light-emitting device based on Er-doped TiO2/p+-Si heterostructure," Applied Physics Letters, vol. 100, Article ID 031103, 2012.

[13] M. Ishihara, S. J. Li, H. Yumoto, K. Akashi, and Y. Ide, "Control of preferential orientation of AlN films prepared by the reactive sputtering method," Thin Solid Films, vol. 316, no. 1-2, pp. 152157, 1998.

[14] M. Ishihara, H. Yumoto, T. Tsuchiya, and K. Akashi, "AlN thin films prepared by the electron shower method," Journal of the Surface Finishing Society of Japan, vol. 47, no. 2, pp. 152-156, 1996.

[15] M. Ishihara, H. Yumoto, T. Tsuchiya, and K. Akashi, "Effect of bias voltage on AlN thin films prepared by electron shower method," Thin Solid Films, vol. 281-282, no. 1-2, pp. 321-323, 1996.

[16] A. Lampert and P. Mark, Current Injection in Solids, Academic, New York, NY, USA, 1970.

[17] S. Fleischer, P. T. Lai, and Y. C. Cheng, "Trap-assisted conduction in nitrided-oxide and re-oxidized nitrided-oxide n-channel metal-oxide-semiconductor field-effect transistors," Journal of Applied Physics, vol. 73, p. 8353, 1994. 
[18] T. Mihara and H. Watanabe, "Electronic conduction characteristics of sol-gel ferroelectric $\mathrm{Pb}(\mathrm{Zr} 0.4 \mathrm{Ti} 0.6) \mathrm{O} 3$ thin-film capacitors: part I," Japanese Journal of Applied Physics, vol. 34, pp. 5664-5673, 1995.

[19] Y. B. Lin and J. Y. Lee, "The temperature dependence of the conduction current in $\mathrm{Ba}_{0.5} \mathrm{Sr}_{0.5} \mathrm{TiO}_{3}$ thin-film capacitors for memory device applications," Journal of Applied Physics, vol. 87, p. 1841, 2000. 

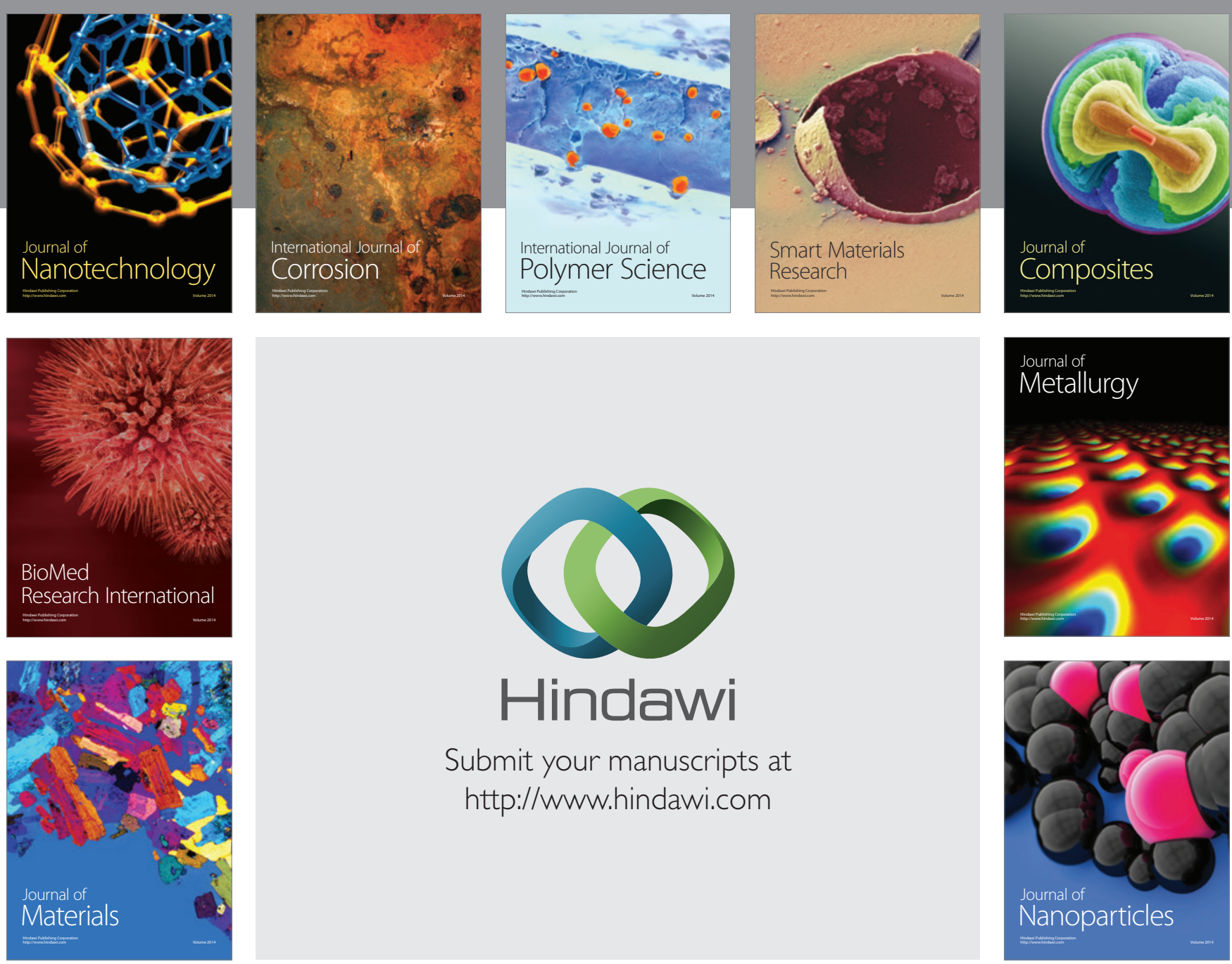

Submit your manuscripts at http://www.hindawi.com
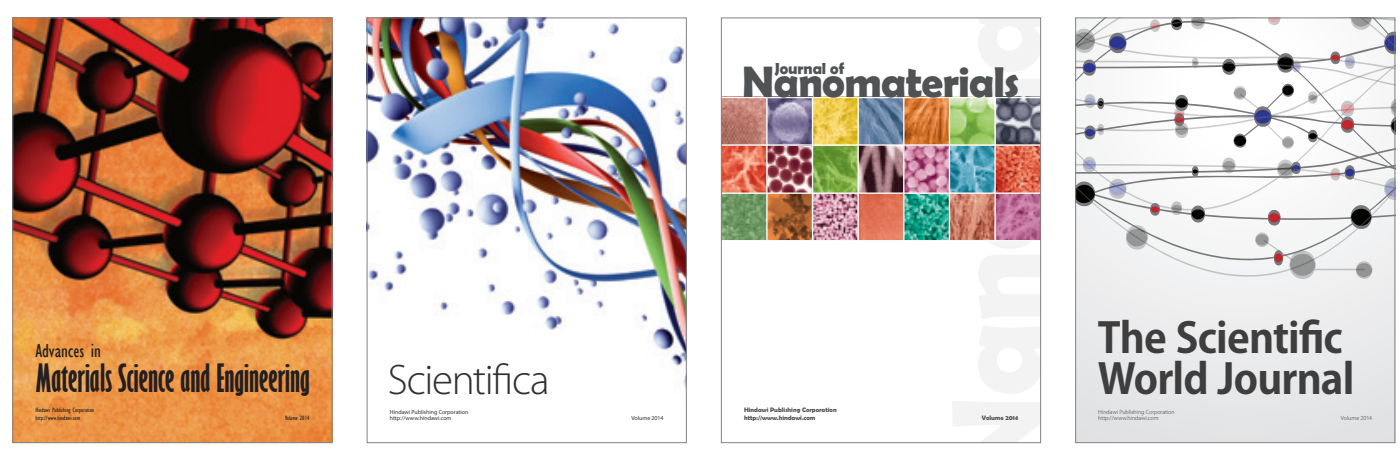

\section{The Scientific World Journal}
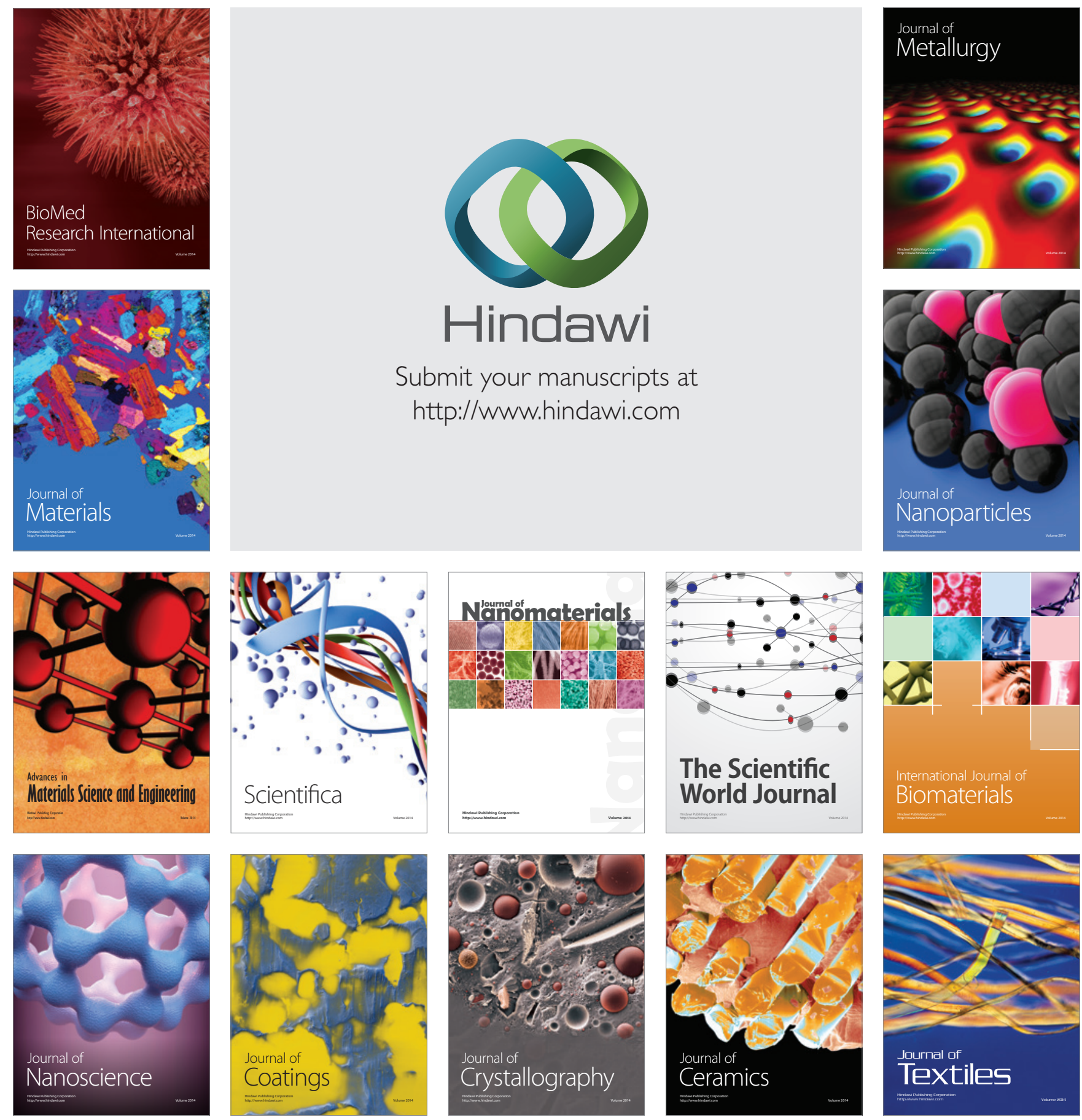